\title{
LOCAL COORDINATES AROUND LIMIT CYCLES OF PARTIAL DIFFERENTIAL EQUATIONS
}

\author{
ARNOLD STOKES
}

\begin{abstract}
Given an isolated periodic solution (limit cycle) $p=p(x, t)$ of a parabolic differential equation in a variable $u=u(x, t)$, local coordinates $(s=s(t), w=w(x, t))$ are introduced so that $w=0, s=t+$ constant corresponds to $u=p$, and the equations for $s, w$ are of the form $d s / d t=1+$ higher-order terms, and $w$ satisfies the variational equation for $p$ on a subspace of codimension one. It is indicated how the method applies to ordinary differential equations, as motivation, and to hyperbolic equations, as an obvious extension.
\end{abstract}

Introduction. Various ways of introducing local coordinates in a neighborhood of a limit cycle of an ordinary differential equation (ODE henceforth) have long been known, see Hartman [4], Hale [2], Urabe [6]. The following note describes a method of introducing local coordinates around a limit cycle (an isolated periodic solution) of a more general equation, a parabolic partial differential equation (PDE). Using these coordinates, one can relate the stability of the limit cycle to the stability behavior of the variational equation on a space of codimension one, just as in ODE. More generally, the study of the stability of weakly coupled systems, each of which oscillate separately, can be facilitated through the use of such coordinate systems. Also, averaging techniques developed for systems with periodic coefficients near an equilibrium point can be extended to systems with a limit cycle by using this coordinate system. And one can discuss the existence of integral manifolds when the system is perturbed, just as in ODE.

$\S 1$ briefly outlines the method as applied to ODE, as this motivates the remainder of this paper. $\$ 2$ then develops the desired coordinates for quasilinear parabolic PDE. At this point it will be clear that one could readily add a $\$ 3$, for hyperbolic PDE, but because the spectral behavior of the period map of linear hyperbolic PDE is much more complicated than that of parabolic PDE, the utility of such coordinates is doubtful, and so $\$ 3$ is omitted. The method of $\$ 1$ can also be extended to functional differential equations (FDE), but due to technical problems, a more subtle approach is needed, which is described in a forthcoming paper, Stokes [5].

Received by the editors October 21, 1975

AMS (MOS) subject classifications (1970). Primary 33B99; Secondary 35K55. 
1. Ordinary differential equations. Consider the equation

$$
\dot{x}=f(x)
$$

and assume it has a periodic solution $p(t)$, of period $2 \pi$. Assume enough differentiability so that, if $A(t)=\partial f(p(t)) / \partial x$, then $\dot{p}(t)$ is a periodic solution of

$$
\dot{y}=A(t) y .
$$

Further, assume there is an adjoint equation

$$
\dot{z}=-z A(t)
$$

with the properties: (a) there is an inner product (or more generally, a bilinear form) so that $z(t) \cdot y(t) \equiv$ constant, where $y, z$ are solutions of (2) and (3) respectively, and (b) (3) has a periodic solution dual to $\dot{p}$, i.e., $v(t) \dot{p}(t) \equiv 1$.

Now introduce local coordinates $(s, h)$ as follows:

$$
x=p(s)+h
$$

(or more precisely, $x(t)=p(s(t))+h(t))$, where we also require

$$
v(s) \cdot h=0 \quad(v(s(t)) \cdot h(t) \equiv 0 \text { for all } t) .
$$

If we write (4) and (5) as a system of equations in $(s, h)$, observe that for $x=p\left(s_{0}\right)$, there is a solution $s=s_{0}, h=0$, with a Jacobian matrix,

$$
\left(\begin{array}{cc}
\dot{p}(s), & I \\
\dot{v}(s) h, & v(s)
\end{array}\right)
$$

which is nonsingular for $h=0$. So there exist unique solutions $(s, h)$ for $x$ near the orbit of $p$.

To determine the differential equations that $s, h$ satisfy if $x(t)$ is a solution of (1), first we need an identity. Differentiating (5), we obtain $\dot{s} \dot{v}(s) h+v(s) \dot{h}$ $\equiv 0$. But $\dot{v}(s)=-v(s) A(s)$, so $v(s)[\dot{h}-\dot{s} A(s) h]=0$, or

$$
v(s)[\dot{h}-A(s) h]=(\dot{s}-1) v(s) A(s) h .
$$

Now, from (4) and (1), $\dot{s} \dot{p}(s)+\dot{h}=f(x)=f(p(s)+h)=f(p(s))+A(s) h$ $+R(s, h)$ (where $R(s, h)=f(p+h)-f(p)-(\partial f(p) / \partial x) h$. As $\dot{p}(s)=f(p(s))$, we obtain

$$
(\dot{s}-1) \dot{p}(s)+h-A(s) h=R(s, h) .
$$

Multiplying by $v$, recalling $v(s) \dot{p}(s)=1$, and using (6), we have $(\dot{s}-1)$ $[1+v(s) A(s) h]=v(s) R(s, h)$, or

$$
\dot{s}=1+\frac{v(s) R(s, h)}{1+v(s) A(s) h}
$$


where $h$ is chosen sufficiently small that $1+v(s) A(s) h>0$. (Note that $v$ and $A$ are periodic, therefore bounded.)

From (7) and (8) it follows directly that

$$
\dot{h}=A(s) h+R(s, h)-\frac{v(s) R(s, h)}{1+v(s) A(s) h} \dot{p}(s)
$$

where $h$, of course, satisfies (5).

Equations (8), (9) and (5) are the equations for $(s, h)$ which are equivalent to (1). Of course, for ODE, one can write $d h / d s=A(s) h+R_{1}(s, h), R_{1}$ secondorder in $h$, and use the condition $v(s) h=0$ and Floquet theory to introduce a variable $w$ of codimension one, $h=Q(s) w$. Then $\dot{w}=B w+R_{2}(s, w)$, and the eigenvalues of the constant matrix $B$ are the $(n-1)$ characteristic exponents of $A$, excluding zero (which corresponds to $\dot{p}$ ). This last equation is the equation usually encountered when introducing local coordinates around a limit cycle in an ODE. But in the general case, where only a partial Floquet theory is available, (8), (9) and (5) seem to be as far as one can go. Of course, for FDE or PDE, one can remove finitely many components from $h$ in (9), and obtain an ODE coupled with an FDE or PDE, but the periodic coefficients cannot be entirely eliminated.

2. Quasi-linear parabolic equations. We consider

$$
u_{t}=L u+f\left(x, u, u_{x}\right), \quad u=0 \text { on } \partial \Omega \times R
$$

where

$$
L u=\sum_{i, j=1}^{n} a_{i j}(x) \frac{\partial^{2} u}{\partial x_{i} \partial x_{j}}
$$

is a uniformly elliptic operator on a domain $\Omega$. Assume the coefficients $\left\{a_{i j}(x)\right\}$ have second derivatives satisfying a uniform $\alpha$-Hölder condition on $\Omega$. Let $p(x, t)$ be a solution of (10), periodic in $t, p=0$ on $\partial \Omega \times R$. Assume $f(x, u, q)$ is $C^{3}(x, u, q)$ for $x \in \Omega,(u, q)$ in some neighborhood of the "orbit" of $p,\left\{\left(p(x, t), p_{x}(x, t)\right): x \in \Omega, t \in R\right\}$.

Let $u=w+p$; then

$$
w_{t}=u_{t}-p_{t}=L(u-p)+f\left(x, w+p, w_{x}+p_{x}\right)-f\left(x, p, p_{x}\right),
$$

or

$$
\begin{gathered}
w_{t}=L w+b w_{x}+c w+R\left(x, t, w, w_{x}\right) \\
\stackrel{\text { def }}{=} L(t) w+R\left(x, t, w, w_{x}\right) .
\end{gathered}
$$

Here

$$
b_{i}=\frac{\partial f}{\partial q_{i}}\left(x, p, p_{x}\right), \quad q_{i}=\frac{\partial u}{\partial x_{i}}, \quad c=\frac{\partial f}{\partial u}\left(x, p, p_{x}\right)
$$


and $R=f\left(x, p+w, p_{x}+w_{x}\right)-f\left(x, p, p_{x}\right)-b w_{x}-c w$. Define $\mathcal{L}(t, w)=\bar{L}(t) w$ $-w_{t}=L w+b w_{x}+c u-u_{t}$. Then the adjoint operator $\mathcal{L}^{*}(t, v)$ is given by $\mathfrak{e}^{*}(t, v)=L v+b^{*} v_{x}+c^{*} v+v_{t}$, where

$$
b_{i}^{*}=-b_{i}+2 \sum_{j} \frac{\partial a_{i j}}{\partial x_{j}}, \quad c^{*}=c-\sum_{i} \frac{\partial b_{i}}{\partial x_{i}}+\sum_{i, j} \frac{\partial^{2} a_{i j}}{\partial x_{i} \partial x_{j}} .
$$

Here we display the argument $t$ in $\bar{L}(t) w, \mathcal{L}(t, w), \mathcal{L}^{*}(t, v)$ to emphasize that the coefficients $b, b^{*}$, etc. depend upon $t$ through the coefficients evaluated at $p(x, t), p_{x}(x, t)$.

In the following, initial functions $w^{0}(x), v^{0}(x)$ will be required to satisfy a uniform $\alpha$-Hölder condition in $\Omega$. Standard existence and uniqueness theorems (see Friedman [8], Edelman [7]) imply that the equation

$$
\ell(t, w)=0, \quad w=0 \quad \text { on } \partial \Omega, \quad w(x, 0)=w^{0}(x)
$$

has a solution $w(x, t)$ defined on $\Omega \times[0, T], T$ the period interval, such that $w_{t}, w_{x}$, and $w_{x x}$ exist and satisfy a uniform $\alpha$-Hölder condition on $\Omega \times[0, T]$. Similar assertions are valid for solutions of $\mathcal{L}^{*}(t, v)=0$.

If $w$ satisfies $\mathcal{L}(t, w)=0$ on $\Omega$, and $v$ satisfies $\mathcal{L}^{*}(t, v)=0$ on $\Omega$, with $w=0=v$ on $\partial \Omega$, then by considering $\int_{\Omega}\left[v \varrho(t, w)-w \mathcal{L}^{*}(t, v)\right] d x$ one concludes $\int_{\Omega} \partial(v w) d x / \partial t=0$. Thus

$$
\int_{\Omega} v(x, t) w(x, t) d x \stackrel{\text { def }}{=}[v(\cdot, t) w(\cdot, t)]=\text { constant } .
$$

Further, one can show that $\mathcal{L}^{*}(t, v)=0$ has a solution $v_{0}(x, t)$ periodic in $t, v_{0}=0$ on $\partial \Omega$, and $\left[v_{0}(\cdot, t), p_{t}(\cdot, t)\right]=1$ (see Gould [1]). Here, of course, $p_{t}$ is periodic solution of $\varrho(t, w)=0$.

Thus the basic structure used in case $I$ is present here also. So introduce local coordinates $s$ and $w(x)$ by

$$
u(x)=p(x, s)+w(x)
$$

with

$$
\left[v_{0}(\cdot, s), w(\cdot)\right]=0 .
$$

Of course $w=0$ on $\partial \Omega$ is still required.

(12) and (13) may be written as

$$
H(s, w)=(0, u)
$$

where $H: R \times C_{\alpha} \rightarrow R \times C_{\alpha}$ is defined by

$$
H(s, w)=\left(\left[v_{0}(\cdot, s), w(\cdot)\right], p(\cdot, s)+w(\cdot)\right) .
$$

Here $C_{\alpha}$ is the space of functions on $\Omega$ satisfying a uniform $\alpha$-Hölder condition, with a norm 


$$
|w|_{\alpha}=\sup \{|w(x)|: x \in \Omega\}+\sup \left\{\left|w(x)-w\left(x^{\prime}\right)\right| /\left|x-x^{\prime}\right|^{\alpha}: x, x^{\prime} \in \Omega\right\} .
$$

Observe that as $p=0$ on $\partial \Omega$, the condition $u=0$ on $\partial \Omega$ implies $w$ $=0$ on $\partial \Omega$, and conversely. To verify that (12), (13) do indeed define a valid coordinate change, it suffices, as in $\S 1$, to appeal to the implicit function theorem. Observe that for $u=p(\cdot, \sigma),(14)$ has a solution $s=\sigma, w=0$. All that remains is to show that $d H(\sigma, 0 ; \cdot, \cdot)$ is a linear homeomorphism on $R \times C_{\alpha}$. Here $d H(\sigma, 0 ; \cdot, \cdot)$ denotes the Fréchet derivative of $H$ on $R \times C_{\alpha}$. Now for $(a, \psi) \in R \times C_{\alpha}, d H(\sigma, 0 ; a, \psi)=d_{1} H(\sigma, 0 ; a)+d_{2} H(\sigma, 0 ; \psi)$ where $d_{1} H=\partial H / \partial s, d_{2} H=\partial H / \partial w$. One sees readily that

$$
d_{1} H(s, w ; a)=\left(a\left[v_{0 s}(\cdot, s), w(\cdot)\right], a p_{t}(\cdot, s)\right),
$$

and

$$
d_{2} H(s, w ; \psi)=\left(\left[v_{0}(\cdot, s), \pi(\cdot)\right], \psi(\cdot)\right)
$$

Thus

$$
d H(\sigma, 0 ; a, \psi)=\left(\left[v_{0}(\cdot, \sigma), \psi(\cdot)\right], a p_{t}(\cdot, \sigma)+\psi(\cdot)\right)
$$

Given $\left(b, \psi_{1}\right) \in R \times C_{\alpha}$, we wish to solve $d H(\sigma, 0 ; a, \psi)=\left(b, \psi_{1}\right)$, or $\left[v_{0}(\cdot, \sigma), \psi(\cdot)\right]=b, a p_{t}(\cdot, \sigma)+\psi(\cdot)=\psi_{1}(\cdot)$. Now $\psi_{1}=a_{1} p_{t}(\cdot, \sigma)+\psi_{2}$, where $\left[v_{0}(\cdot, \sigma), \psi_{1}\right]=a_{1},\left[v_{0}(\cdot, \sigma), \psi_{2}\right]=0$. So the desired pair $(a, \psi)$ is given by $a=a_{1}-b, \psi(\cdot)=\psi_{2}(\cdot)+b p_{t}(\cdot, \sigma)$. Clearly $a_{1}$ depends continuously on $\psi_{1}$, and so $(a, \psi)$ depends continuously on $\left(b, \psi_{1}\right)$. So (14) has a unique solution in some neighborhood of $p(\cdot, \sigma)$ for each $\sigma$, and this solution is continuous and differentiable in $u$ (as $H$ is differentiable in $(s, w)$ ).

The compactness of the orbit of $p$ implies that the above solution is in fact defined on some neighborhood of the orbit of $p$, which establishes the validity of the new coordinates $(s, w)$ introduced in (12) and (13).

From (10), it follows that

$$
\dot{s} p_{t}(x, s)+w_{t}(x, t)=L(p+w)+f(x, p(x, s)+w(x, t)) .
$$

As $p(x, s)$ is a solution of $(10)$, it follows that

$$
(s-1) p_{t}(x, s)+w_{t}(x, t)=\bar{L}(s) w+R\left(x, s, w, w_{x}\right) .
$$

Again an identity must be derived, by differentiating (13) with respect to $t$. From (13) it follows that

$$
0=(s(t)-1) \int_{\Omega} v_{0 t}(x, s) w(x, t) d x+\int_{\Omega}\left(v_{0}(x, s) w_{t}(x, t)+v_{0 t}(x, s) w(x, t)\right) d x .
$$




$$
\begin{gathered}
v_{0}(x, s) w_{t}(x, t)+v_{0 t}(x, s) w(x, t) \\
=\sum_{i} \frac{\partial}{\partial x_{i}}\left\{\sum _ { j } \left[v_{0}(x, s) a_{i j}(x) \frac{\partial w}{\partial x_{j}}(x, t)-w(x, t) a_{i j}(x) \frac{\partial v_{0}}{\partial x_{j}}(x, s)\right.\right. \\
\left.-w(x, t) v_{0}(x, s) \frac{\partial a_{i j}(x)}{\partial x_{j}}\right] \\
\left.+b_{i}(x, s) w(x, t) v_{0}(x, s)\right\} \\
+\mathcal{L}^{*}\left(s, v_{0}(x, s)\right) w(x, t)-v_{0}(x, s) \mathcal{L}(s, w(x, t)) .
\end{gathered}
$$

But using the divergence theorem, the boundary conditions on $w$ and $v_{0}$, together with $\mathfrak{L}^{*}\left(s, v_{0}(x, s)\right)=0$, one obtains

$$
(s-1)\left[v_{0 t}(\cdot, s), w(\cdot, t)\right]=\left[v_{0}(\cdot, s), \mathcal{L}(s, w(\cdot, t))\right] .
$$

Equation (15) can be rewritten as

$$
(\dot{s}-1) p_{t}(x, s)-\mathcal{L}(s, w(x, t))=R\left(x, s, w, w_{x}\right) .
$$

Taking the inner product of both sides with $v_{0}(x, s)$, and using (16), it results that

$$
(\dot{s}-1)\left(1-\left[v_{0 t}(\cdot, s), w(\cdot, t)\right]\right)=\left[v_{0}(\cdot, s), R\left(\cdot, s, w, w_{x}\right)\right] .
$$

Now $v_{0}$ periodic in $t$ implies $v_{0}$ bounded, and restricting $w$ in norm so that $\left[v_{0 t}(\cdot, s), w(\cdot, t)\right]<1$, we obtain

$$
\dot{s}(t)=1+\frac{\left[v_{0}(\cdot, s), R\left(\cdot, s, w, w_{x}\right)\right]}{1-\left[v_{0 t}(\cdot, s), w(\cdot, t)\right]}=1+R_{1}\left(s, w, w_{x}\right) .
$$

From (18), one can express $\dot{s}-1$ as a second-order term in $w$. Introducing this expression in (15) gives

$$
w_{t}(x, t)=\bar{L}(s) w+R\left(x, s, w, w_{x}\right)-R_{1}\left(s, w, w_{x}\right) p_{t}(x, s) .
$$

Thus we have proved the following theorem:

THEOREM. If $f \in C^{3}\left(x, u, u_{x}\right)$ on $\Omega$, then the transformation of coordinates given by

$$
u(x, t)=p(x, s(t))+w(x, t)
$$

transforms (10) into an equivalent system,

$$
\begin{aligned}
\dot{s}(t) & =1+\frac{\left[v_{0}(\cdot, s), R\left(\cdot, s, w, w_{x}\right)\right]}{1-\left[v_{0 t}(\cdot, s), w(\cdot, t)\right]} \stackrel{\text { def }}{=} 1+R_{1}\left(s, w, w_{x}\right), \\
w_{t}(x, t) & =\bar{L}(s) w+R\left(x, s, w, w_{x}\right)-R_{1}\left(s, w, w_{x}\right) p_{t}(x, s)
\end{aligned}
$$


where $w$ satisfies

$$
\left[v_{0}(\cdot, s), w(\cdot, t)\right]=0 .
$$

Here $\bar{L}(s) w$ and $R$ are defined in (11), and $R$ is second-order in $w$, for $w$ near 0 , in a Hölder-norm. The transformation (20) is valid in some neighborhood of the orbit of $p$.

REMARK 1. Note that the variable $s$ is independent of $x$, as is clear from (21), so letting $\bar{w}(x, s)=w(x, t(s))$, we can eliminate the variable $t$, and then $\bar{w}$ satisfies (23), and an equation of the form

$$
\bar{w}_{s}(x, s)=\bar{L}(s) w+R_{2}\left(s, \bar{w}, \bar{w}_{x}\right)
$$

where $R_{2}$ is second-order in $w$, for $w$ near zero.

REMARK 2. As mentioned earlier, a finite number of components of $\bar{w}$ may be singled out in (24), corresponding to a finite number of the characteristic multipliers of the period map of $\varrho(s, \bar{w})=0$. The procedure is identical with that outlined in Hale [3, p. 120], for FDE. The result is a pair of coupled equations, one an ODE, the second similar to (24), but restricted to a subspace of finite codimension.

\section{REFERENCES}

1. P. Gould, Oscillations in nonlinear parabolic systems, Ph.D. thesis, Georgetown Univ., Washington, D.C., 1972.

2. J. K. Hale, Orindary differential equations, Interscience, New York, 1969.

3. _-, Functional differential equations, Springer-Verlag, New York, 1971.

4. P. Hartman, Ordinary differential equations, Wiley, New York, 1964. MR 30 \#1270.

5. A. Stokes, Local coordinates around a limit cycle of a functional differential equation with applications, J. Differential Equations (to appear).

6. M. Urabe, Geometric study of nonlinear autonomous oscillations, Funkcial. Ekvac. 1 (1958), 1-84. MR 20 \#5921.

7. S. D. Ėìdel'man, Parabolic systems, "Nauka", Moscow, 1964; English transl., Scripta Technica, London; North-Holland, Amsterdam; Noordhoff, Groningen, 1969. MR 29 \#4998; 40 \#6023.

8. A. Friedman, Partial differential equations of parabolic type, Prentice-Hall, Englewood Cliffs, N.J., 1964. MR 31 \#6062.

Department of Mathematics, Georgetown University, Washington, D.C. 20057 